\title{
Rational invariants of scalings from Hermite normal forms.
}

\author{
Evelyne Hubert *
}

\begin{abstract}
Scalings form a class of group actions on affine spaces that have both theoretical and practical importance. A scaling is accurately described by an integer matrix. Tools from linear algebra are exploited to compute a minimal generating set of rational invariants, trivial rewriting and rational sections for such a group action. The primary tools used are Hermite normal forms and their unimodular multipliers. With the same line of ideas, a complete solution to the scaling symmetry reduction of a polynomial system is also presented.
\end{abstract}

Keywords: Matrix normal form; Group actions; Rational invariants; Symmetry reduction.

AMS Classification: 08-04 12-04 14L30 15-04

\section{INTRODUCTION}

Scalings form a simple class of group actions: they are diagonal actions of a torus on an affine space. For example,

$\left[(\mu, \nu),\left(z_{1}, z_{2}, z_{3}, z_{4}, z_{5}\right)\right] \rightarrow\left(\mu^{6} z_{1}, \nu^{3} z_{2}, \frac{\nu}{\mu^{4}} z_{3}, \frac{\mu}{\nu^{4}} z_{4}, \mu^{3} \nu^{3} z_{5}\right)$

describes the action of the group $\left(\mathbb{R}^{*}\right)^{2}$, with coordinates $(\mu, \nu)$, on $\mathbb{R}^{5}$, with coordinates $\left(z_{1}, z_{2}, z_{3}, z_{4}, z_{5}\right)$. The action simply rescales each individual coordinate. One can check that the three rational functions

$$
g_{1}=z_{1} z_{2}^{2} z_{3}^{2} z_{4}^{2}, \quad g_{2}=\frac{z_{2}^{3} z_{4}^{2}}{z_{1} z_{3}}, \quad g_{3}=\frac{z_{2} z_{4} z_{5}}{z_{1}^{2} z_{3}^{2}}
$$

are left invariant by any of the above transformation determined by $(\mu, \nu)$. They actually form a generating set of invariants of the scaling: they have the property that any other rational invariant $f$ can be written as a rational function of them. In fact they have an even stronger property: the rewriting is given by a simple substitution. Indeed, if

*INRIA Méditerranée, France. Evelyne.Hubert@inria.fr ${ }^{\dagger}$ Cheriton School of Computer Science, University of Waterloo, Waterloo ON, Canada N2L 3G1 glabahn@uwaterloo.ca

\author{
George Labahn †
}

$f(z)$ is a rational invariant then

$$
f\left(z_{1}, z_{2}, z_{3}, z_{4}, z_{5}\right)=f\left(g_{1}^{-1}, g_{2}, g_{1}, g_{2}^{-1}, g_{3}\right) .
$$

Providing a generating set of rational invariants along with an associated rewriting substitution for any given scaling is the first goal of the present article.

Though simple, scalings and their invariants have considerable practical importance. On the theoretical front scalings are known as torus actions and play a major role in algebraic geometry and combinatorics. Besides they underlie what is known as dimensional analysis with the invariants giving the dimensionless quantities needed to derive physical laws $[2,3,10]$. Dimensional analysis has been automated in the works [11] and [13]. Central to this is the Buckingham- $\pi$ theorem. A reinterpretation of it states that a fundamental set of invariants is obtained from the basis of the nullspace of a matrix of exponents of the scaling [19, Section 3.4]. A second use of scalings is that they give mathematical sense to the rule of thumb used to reduce the number of parameters in biological models $[18,15]$. This reduction by scaling symmetry of dynamical or polynomial systems was previously studied in [9, 14, 23].

In this paper we go further in this direction than handled in the previous cited works. In particular we produce invariants which are rational functions, that is which do not involve any square roots or other fractional powers of the variables. In addition we provide trivial rewrite rules for our generating set of invariants. By this we mean that we give explicit substitution rules for rewriting any rational invariant (and actually any smooth invariant) in terms of the generating set. Again, this operation is performed without introducing any radicals.

Algorithmic tools for finding generating rational invariants and rewrite rules for the general class of rational actions of an algebraic group typically require Gröbner bases computations $[17,7,12]$. A rewriting substitution can be achieved provided we allow algebraic functions [8].

In the case of scaling we show that a unimodular multiplier for the Hermite normal form of the integer matrix of exponents contains even further information. The unimodular multiplier provides a basis for the integer lattice of vectors in the kernel of the matrix of exponents. This basis actually describes rational invariant given as Laurent monomials (that is, monomials where we allow negative powers). We 
show that these invariants form a generating set for the field of rational invariants. It is furthermore a minimal such set. In fact we show more than the generation property. We also provide a simple method to rewrite any invariant in terms of these monomials via variable substitution. The substitution is read off from the inverse of the unimodular multiplier.

The triviality of the rewrite rules actually reflects the existence of a rational section to the orbits of the action. The equation of the section can be read off the unimodular multiplier, something of independent theoretical interest in the area of group actions. The unimodular multiplier for the Hermite form of the matrix of exponents is not unique. We give a construction for a canonical unimodular multipler which allows us to pinpoint the simplest rational sections.

In order to show a practical application of the new tools offered we address and solve a symmetry reduction problem. The knowledge of some symmetry of the solution set of a polynomial system brings hope that we can reduce the size of the problem by factoring out the symmetry. With a generating set and rewrite rules at hand we can indeed write the reduced system. The new variables are the generating invariants. We have here a minimal set of those and thus the number of variabes and the dimension of the solution set is exactly reduced by the dimension of the group. In the symmetry reduction business, a more subtle task is actually to retrieve the solution of the original system from the solution of the reduced system. In the case of scaling we show how to parameterize all the toric solutions of the original system from the solutions of the reduced system.

\section{INTEGER MATRIX NORMAL FORMS}

In this section we provide the basic information about the Hermite normal form of a matrix of integers and its unimodular multiplier. We propose a canonical unimodular multiplier that is relevant in providing a simple rational section to the orbits of a scaling.

\subsection{Hermite Normal Forms}

Definition 2.1 An $m \times n$ integer matrix $H=\left[h_{i j}\right]$ is in column Hermite Normal Form if there exists an integer $r$ and a strictly increasing sequence $i_{1}<i_{2}<\cdots<i_{r}$ of pivot rows such that

(i) The first $r$ columns are nonzero;

(ii) $h_{k, j}=0$ for $k>i_{j}$;

(iii) $0 \leq h_{i_{j}, k}<h_{i_{j}, j}$ when $j<k$.

Thus a matrix is in column Hermite normal form if the submatrix formed by the pivot rows $i_{1}, \cdots, i_{r}$ and the first $r$ columns is upper triangular and that all nonzero elements of the pivot rows are positive and less than the corresponding (positive) diagonal entry. The integer $r$ is the rank of the matrix. By changing column to row and row to column indices in (ii) and (iii) one obtains the row Hermite Normal Form of a matrix of integers.

Every integer matrix can be transformed via integer column operations to obtain a unique column Hermite form. The column operations are encoded in unimodular matrices, that is, invertible integer matrices whose inverses are also integer matrices. Thus for each $A$ there exists a unimodular matrix $V$ such that $A \cdot V$ is in Hermite normal form. Similar statements also hold for the row Hermite normal form. We refer the reader to $[4,22]$ for more information on such forms.

When $A \in \mathbb{Z}^{r \times n}$, with $r \leq n$, has full row rank $r$ then there exists a unimodular matrix $V$ such that

$$
A \cdot V=[H, 0] \text { with } H \in \mathbb{Z}^{r \times r} \text { of full rank. }
$$

If $W \in \mathbb{Z}^{n \times n}$ is the inverse of $V$ then we can partition $V$ and $W$ as

$$
V=\left[V_{\mathfrak{i}}, V_{\mathfrak{n}}\right] \text { with } V_{\mathfrak{i}} \in \mathbb{Z}^{n \times r} \text { and } V_{\mathfrak{n}} \in \mathbb{Z}^{n \times(n-r)}
$$

and

$$
W=\left[\begin{array}{l}
W_{\mathfrak{u}} \\
W_{\mathfrak{o}}
\end{array}\right] \text { with } W_{\mathfrak{u}} \in \mathbb{Z}^{r \times n} \text { and } W_{\mathfrak{o}} \in \mathbb{Z}^{(n-r) \times n} .
$$

We then have

$$
\begin{aligned}
& I_{n}=W V=\left[\begin{array}{ll}
W_{\mathfrak{u}} V_{\mathfrak{i}} & W_{\mathfrak{u}} V_{\mathfrak{n}} \\
W_{\mathfrak{o}} V_{\mathfrak{i}} & W_{\mathfrak{d}} V_{\mathfrak{n}}
\end{array}\right] \\
& I_{n}=V W=V_{\mathfrak{i}} W_{\mathfrak{u}}+V_{\mathfrak{n}} W_{\mathfrak{o}} .
\end{aligned}
$$

Note that the blocks of $V$ provide the Hermite normalization of the blocks of $W$ since from (4) we have

$$
W_{\mathfrak{u}}\left[V_{\mathfrak{i}}, V_{\mathfrak{n}}\right]=\left[I_{r}, 0\right] \quad \text { and } \quad W_{\mathfrak{d}}\left[V_{\mathfrak{n}}, V_{\mathfrak{i}}\right]=\left[I_{n-r}, 0\right] .
$$

We state a known properties of Hermite normal forms [4, $22]$ in a way that is needed later in the paper.

Lemma 2.2 Let $A \in \mathbb{Z}^{r \times n}$ be a full row rank matrix and $V \in \mathbb{Z}^{n \times n}$ a unimodular matrix such that $A V=[H, 0]$ with $H \in \mathbb{Z}^{r \times r}$. If $V$ is partitioned as in (2), then the columns of $V_{\mathfrak{n}}$ form a basis for the integer lattice defined by the kernel of $A$.

\subsection{Normal unimodular multiplier}

For the problem of interest in this paper the number of columns is larger than the rank. In this case the unimodular multiplier is not unique. Indeed, with the partition $V=\left[V_{\mathfrak{i}}, V_{\mathfrak{n}}\right]$ as in (2), any column operation using the columns of $V_{\mathfrak{n}}$ do not affect the Hermite form $H$ for the initial matrix $A$ and hence results in a different unimodular multiplier $V$. In this subsection we describe a normalization of the multiplier $V$ which is both simple and unique. Previous work on determining unique unimodular multipliers includes that of [6] for integer matrices where the unimodular multiplier is reduced via lattice reduction. We favor the component $V_{\mathfrak{n}}$ to be in Hermite normal form, as in [1], which deals with polynomial matrices. We indeed prefer that the component $V_{\mathfrak{n}}$ be in The triangular form obtained is useful for our application.

Theorem 2.3 Suppose $A \in \mathbb{Z}^{r \times n}$ has full row rank. Then there exists a unique unimodular matrix $V$ such that

(a) $A \cdot V=[H, 0]$ with $H \in \mathbb{Z}^{r \times n}$ in column Hermite normal form, 
(b) $V=\left[V_{\mathfrak{i}}, V_{\mathfrak{n}}\right]$ with $V_{\mathfrak{n}} \in \mathbb{Z}^{n \times(n-r)}$ in column Hermite normal form,

(c) If $i_{1}<i_{2}<\cdots<i_{n-r}$ are the pivots rows for $V_{\mathfrak{n}}$ then for each $1 \leq j \leq n-r$ :

$$
0 \leq\left[V_{\mathfrak{i}}\right]_{i_{j}, k}<\left[V_{\mathfrak{n}}\right]_{i_{j}, j} \text { for all } 1 \leq k \leq r .
$$

That is, $V_{\mathrm{i}}$ is reduced with respect to the pivots rows of $V_{\mathrm{n}}$.

Proof. The existence of a unimodular matrix $V$ satisfying (a) and (b) follows directly from the existence of column Hermite forms. The reduction (c) follows by doing the column operation

$\left[V_{\mathrm{i}}\right]_{i_{j}, k} \leftarrow\left[V_{\mathbf{i}}\right]_{i_{j}, k}-q \cdot\left[V_{\mathfrak{n}}\right]_{i_{j}, j}$ with $q=\operatorname{iquo}\left(\left[V_{\mathbf{i}}\right]_{i_{j}, k},\left[V_{\mathfrak{n}}\right]_{i_{j}, j}\right)$

for each $k$ as $j$ varies from column $n-r$ to 1 . Here iquo denotes integer quotient, a function which always results in a nonnegative remainder.

It remains to show that any $V$ satisfying (a), (b) and (c) is unique. Thus we suppose the contrary and assume that we have $A \cdot V=A \cdot V^{*}=[H, 0]$ with $V=\left[V_{\mathfrak{i}}, V_{\mathfrak{n}}\right]$ and $V^{*}=\left[V_{\mathfrak{i}}^{*}, V_{\mathfrak{n}}^{*}\right]$ both being unimodular and satisfying (a), (b) and (c). Since both $V_{\mathfrak{n}}$ and $V_{\mathfrak{n}}^{*}$ form a basis for the kernel (over $\mathbb{Z}$ ) of $A$ there exists an integer matrix $U^{*}$ such that $V_{\mathfrak{n}}=V_{\mathfrak{n}}^{*} \cdot U^{*}$. The uniqueness of column Hermite forms then implies that $U^{*}=I$ and so $V_{\mathfrak{n}}=V_{\mathfrak{n}}^{*}$.

Finally, in order to show $V_{\mathrm{i}}=V_{\mathrm{i}}^{*}$ we first notice that $V_{\mathrm{i}}-V_{\mathrm{i}}^{*}$ is in the kernel of $A$. Since the columns of $V_{\mathfrak{n}}$ form a basis for this kernel, there exists an integer matrix $U$ such that $V_{\mathrm{i}}-V_{\mathrm{i}}^{*}=V_{\mathfrak{n}} \cdot U$. Looking at the last pivot row of $V_{\mathfrak{n}}$ (row $i_{n-r}$ ) and using condition (c) we have that for each $1 \leq k \leq r$ :

$$
\left[V_{\mathrm{i}}\right]_{i_{n-r}, k}-\left[V_{\mathrm{i}}^{*}\right]_{i_{n-r}, k}=\left[V_{\mathfrak{n}}\right]_{i_{n-r}, n-r} \cdot u_{n-r, k} .
$$

From condition (c) we have that both $\left[V_{\mathrm{i}}\right]_{i_{n-r}, k}$ and $\left[V_{\mathrm{i}}^{*}\right]_{i_{n-r}, k}$ are positive integers smaller than $\left[V_{\mathfrak{n}}\right]_{i_{n-r}, n-r}$. Thus $u_{n-r, k}=$ 0 for all $k$ and hence the last row of $U$ is zero. Suppose now that rows $n-r, \ldots, \ell+1$ of $U$ are all zero. Then for the pivot row $i_{\ell}$ the triangular property of the Hermite form implies that for each $k$ we have

$$
\left[V_{\mathrm{i}}\right]_{i_{\ell}, k}-\left[V_{\mathrm{i}}^{*}\right]_{i_{\ell}, k}=\left[V_{\mathfrak{n}}\right]_{i_{\ell}, \ell} \cdot u_{\ell, k} .
$$

As before, the size condition (c) implies that $u_{\ell, k}=0$ for all $k$ and hence row $\ell$ of $U$ is zero. By induction we see that $U=0$. Hence $V_{\mathrm{i}}=V_{\mathrm{i}}^{*}$ and so $V=V^{*}$ is unique.

\section{Example 2.4 Let}

$$
A=\left[\begin{array}{ccccc}
8 & 2 & 15 & 9 & 11 \\
6 & 0 & 6 & 2 & 3
\end{array}\right]
$$

which has Hermite normal form $\left[I_{2}, 0\right]$. The reduction performed by Maple results in the unimodular multiplier

$$
V^{\prime}=\left[\begin{array}{rrrrr}
-49 & -1 & -57 & -13 & -28 \\
-36 & -1 & -42 & -10 & -21 \\
79 & 2 & 92 & 21 & 45 \\
-36 & -1 & -42 & -9 & -21 \\
-36 & -1 & -42 & -10 & -20
\end{array}\right]
$$

while the normalized unimodular multiplier is

$$
V=\left[\begin{array}{rrrrr}
-1 & -2 & -2 & -2 & -1 \\
-3 & -14 & -7 & -13 & -7 \\
1 & 1 & 2 & 1 & 0 \\
0 & 2 & 0 & 3 & 0 \\
0 & 1 & 0 & 0 & 2
\end{array}\right]
$$

\section{SCALINGS}

Scalings can be described through the matrix of exponents of the group parameters as they act on each component. Similar descriptions are used for toric ideals [24]. In this section we describe the matrix forms and properties that are useful when representing scalings and computing their invariants.

\subsection{Matrix notations for monomial maps}

If $a=\left[a_{1}, \ldots, a_{r}\right]^{T}$ is a column vector of integers and $\lambda=$ $\left[\lambda_{1}, \ldots, \lambda_{r}\right]$ is a row vector with entries in $\mathbb{K}^{*}$, then $\lambda^{a}$ denotes the scalar

$$
\lambda^{a}=\lambda_{1}^{a_{1}} \cdots \lambda_{r}^{a_{r}} .
$$

If $\lambda=\left[\lambda_{1}, \ldots, \lambda_{r}\right]$ is a row vector of $r$ indeterminates, then $\lambda^{a}$ can be understood as a monomial in the Laurent polynomial ring $\mathbb{K}\left[\lambda, \lambda^{-1}\right]$, a domain isomorphic to $\mathbb{K}[\lambda, \mu] /\left(\lambda_{1} \mu_{1}-\right.$ $\left.1, \ldots, \lambda_{r} \mu_{r}-1\right)$. We extend this notation to matrices: If $A$ is an $r \times n$ matrix then $\lambda^{A}$ is the row vector

$$
\lambda^{A}=\left[\lambda^{A \cdot, 1}, \cdots, \lambda^{A \cdot, n}\right]
$$

where $A_{\cdot, 1}, \ldots, A_{\cdot, n}$ are the $n$ columns of $A$.

If $x=\left[x_{1}, \ldots, x_{n}\right]$ and $y=\left[y_{1}, \ldots, y_{n}\right]$ are two row vectors, we write $x \star y$ for the row vector obtained by component wise multiplication:

$$
x \star y=\left[x_{1} y_{1}, \ldots, x_{n} y_{n}\right]
$$

Proposition 3.1 Suppose $A$ and $B$ are matrices of size $r \times$ $n$ and $n \times n$, respectively, and that $\lambda$ is a row vector with $r$ components. Then

(a) If $A=\left[A_{\mathfrak{i}}, A_{\mathfrak{n}}\right]$ is a partition of the columns of $A$, then $\lambda^{A}=\left[\lambda^{A_{\mathfrak{i}}}, \lambda^{A_{\mathfrak{n}}}\right]$,

(b) $\lambda^{A B}=\left(\lambda^{A}\right)^{B}$

(c) $(y \star z)^{A}=y^{A} \star z^{A}$.

(d) $\lambda^{A+B}=\lambda^{A} \star \lambda^{B}$

Proof. Part (a) follows directly from the definition of $\lambda^{A}$. For part (b) we have for each component $j, 1 \leq j \leq t$ :

$$
\begin{aligned}
{\left[\left(\lambda^{A}\right)^{B}\right]_{j} } & =\prod_{i=1}^{n}\left[\lambda^{A}\right]_{i}^{b_{i j}} \\
& =\prod_{i=1}^{n}\left(\prod_{\ell=1}^{r} \lambda_{\ell}^{a_{\ell i}}\right)^{b_{i j}} \\
& =\prod_{\ell=1}^{r}\left(\prod_{i=1}^{n} \lambda_{\ell}^{a_{\ell i} b_{i j}}\right) \\
& =\prod_{\ell=1}^{r}\left(\lambda_{\ell}^{\sum_{i=1}^{n} a_{\ell i} b_{i j}}\right)=\left[\lambda^{A B}\right]_{j} .
\end{aligned}
$$

For part (c) one simply notices that for each $j$ we have

$$
\begin{aligned}
{\left[(y \star z)^{A}\right]_{j} } & =\prod_{i}^{r}[y \star z]_{i}^{a_{i, j}}=\prod_{i}^{r} y_{i}^{a_{i, j}} \cdot z_{i}^{a_{i, j}} \\
& =\left[y^{A}\right]_{j}\left[z^{A}\right]_{j}=\left[y^{A} \star z^{A}\right]_{j} .
\end{aligned}
$$

The proof of $(\mathrm{d})$ follows along the same lines. 


\subsection{Scalings in matrix notation}

We consider an algebraically closed field $\mathbb{K}$ of characteristic zero, the multiplicative group of which is $\mathbb{K}^{*}$. The $r$ dimensional torus is the Abelian group $\left(\mathbb{K}^{*}\right)^{r}$. Its identity is $1_{r}=(1, \ldots, 1)$ and the group operation is componentwise multiplication, which we denoted $\star$.

Definition 3.2 Let $A$ be a $r \times n$ integer matrix: $A \in \mathbb{Z}^{r \times n}$. The associated scaling is the linear action of $T=\left(\mathbb{K}^{*}\right)^{r}$ on the affine space $\mathbb{K}^{n}$ given by

$$
\begin{aligned}
\left(\mathbb{K}^{*}\right)^{r} \times \mathbb{K}^{n} & \rightarrow \mathbb{K}^{n} \\
(\lambda, \quad z) & \rightarrow \lambda^{A} \star z .
\end{aligned}
$$

With the notations introduced above we have that

$$
\lambda^{A} \star z=\left[\lambda^{A_{\cdot, 1}} z_{1}, \ldots, \lambda^{A \cdot, n} z_{n}\right]
$$

with $A_{\cdot, 1}, \ldots, A_{\cdot, n}$ being the $n$ columns of $A$. Thus for each $j=1, \ldots, n$ the action scales the $j^{t h}$ component $z_{j}$ by the power product $\lambda_{1}^{a_{1, j}} \cdots \lambda_{r}^{a_{r, j}}$. The axioms for a group action are satisfied thanks to Proposition 3.1: $1_{r} \star z=z$ and $(\lambda \star$ $\mu)^{A} \star z=\lambda^{A} \star\left(\mu^{A} \star z\right)$.

There is no loss of generality in assuming that $A$ has full row rank. Indeed, we can view the scaling defined by $A$ as a diagonal representation of $\left(\mathbb{K}^{*}\right)^{r}$ on the $n$ dimensional space $\mathbb{K}^{n}$

$$
\begin{array}{ccc}
\left(\mathbb{K}^{*}\right)^{r} & \rightarrow & \mathrm{D}_{n} \\
\left(\lambda_{1}, \ldots, \lambda_{r}\right) & \mapsto \operatorname{diag}\left(\lambda^{A}\right)
\end{array}
$$

where $\mathrm{D}_{n}$ is the group of invertible diagonal matrices. This in turn can be factored by the group morphism from $\left(\mathbb{K}^{*}\right)^{r}$ to $\left(\mathbb{K}^{*}\right)^{n}$ defined by $A$. This is given explicitly by:

$$
\begin{aligned}
& \rho(A): \quad\left(\mathbb{K}^{*}\right)^{r} \quad \rightarrow\left(\mathbb{K}^{*}\right)^{n} \\
& \left(\lambda_{1}, \ldots, \lambda_{r}\right) \mapsto \quad \lambda^{A}
\end{aligned}
$$

Suppose now that $U A=\left[\begin{array}{c}B \\ 0\end{array}\right]$ is a row Hermite form for $A$ with unimodular row multiplier $U$. Writing $U=\left[\begin{array}{c}U_{1} \\ U_{2}\end{array}\right]$ where $U_{1} A=B$ is of row dimension $d$ we have that $U_{2} A=0$. Then

$$
\begin{array}{clccc}
\left(\mathbb{K}^{*}\right)^{d} \times\left(\mathbb{K}^{*}\right)^{r-d} & \stackrel{U}{\rightarrow} & \left(\mathbb{K}^{*}\right)^{r} & \stackrel{A}{\rightarrow} & \left(\mathbb{K}^{*}\right)^{n} \\
\left(\mu_{1}, \mu_{2}\right) & \mapsto & \mu_{1}^{U_{1}} \star \mu_{2}^{U_{2}} & \mapsto & \left(\mu_{1}^{U_{1}} \star \mu_{2}^{U_{2}}\right)^{A}=\mu_{1}^{B} .
\end{array}
$$

Since $U$ is unimodular, $\rho(U)$ is an isomorphism of groups and the image of $\left(\mathbb{K}^{*}\right)^{r}$ by $\rho(A)$ is equal to the image of $\left(\mathbb{K}^{*}\right)^{d}$ by $\rho(B)$.

\section{RATIONAL INVARIANTS}

Consider a full row rank matrix $A \in \mathbb{Z}^{r \times n}$ which defines an action of the torus $T=\left(\mathbb{K}^{*}\right)^{r}$ on $\mathbb{K}^{n}$. A rational invariant is an element $f$ of $\mathbb{K}(z)$ such that $f\left(\lambda^{A} \star z\right)=f(z)$. Rational invariants form the subfield $\mathbb{K}(z)^{T}$ of $\mathbb{K}(z)$. In this section we show how a unimodular multiplier $V$, where $A \cdot V$ is the Hermite normal form, provides us with a complete description of the subfield of rational invariants. From $V$ we shall extract
- $n-r$ generating rational invariants, which are actually Laurent monomials,

- a simple rewriting of any rational invariant in terms of this generating set,

- a rational section to the orbits of the scaling.

We thus go much further than the group action transcription of the Buckingham $\pi$-theorem of dimensional analysis $[2,19]$. This latter takes any basis of the nullspace of the matrix $A$ and provides a set of functionally generating invariants, some of which could involve fractional powers. In the present approach, only integer powers are involved. This spares us the determination of proper domains of definition. Furthermore, the Buckingham $\pi$-theorem gives no indication on how to rewrite an invariant in terms of the generators produced. The rewriting we propose is a simple substitution. This is reminiscent of the normalized invariants appearing in $[5,8,16]$ (or replacement invariants in [7]). And indeed, using the terminology of those articles, we are in a position to exhibit a global cross-section (of degree one) to the orbits of the scaling. Note though, that the substitution is again rational: we do not introduce any algebraic functions.

\subsection{Generating and replacement invariants}

A Laurent monomial $z^{v}$ is a rational invariant if $\left(\lambda^{A} \star z\right)^{v}=$ $z^{v}$ and therefore if and only if $A v=0$. The following theorem shows that rational invariants of a scaling can be written as a rational function of Laurent monomials that are invariants.

Lemma 4.1 Suppose $\frac{p}{q} \in \mathbb{K}(z)^{T}$, with $p, q \in \mathbb{K}[z]$ relatively prime. Then there exists $u \in \mathbb{Z}^{n}$ such that

$$
p(z)=\sum_{v \in \operatorname{ker} A \cap \mathbb{Z}^{n}} a_{v} z^{u+v} \text { and } q(z)=\sum_{v \in \operatorname{ker} A \cap \mathbb{Z}^{n}} b_{v} z^{u+v}
$$

where the families of coefficients, $\left(a_{v}\right)_{v}$ and $\left(b_{v}\right)_{v}$, have finite support. $^{1}$

Proof. We take advantage of the more general fact that rational invariants of a linear action on $\mathbb{K}^{n}$ are quotients of semi-invariants (see for instance [21, Theorem 3.3]). Indeed, if $p / q$ is a rational invariant, then we have

$$
p(z) q\left(\lambda^{A} \star z\right)=p\left(\lambda^{A} \star z\right) q(z)
$$

in $\mathbb{K}(\lambda)[z]$. As $p$ and $q$ are relatively prime, $p(z)$ divides $p\left(\lambda^{A} \star z\right)$ and, since these two polynomials have the same degree, there exists $\chi(\lambda) \in \mathbb{K}(\lambda)$ such that $p\left(\lambda^{A} \star z\right)=$ $\chi(\lambda) p(z)$. It then also follows that $q\left(\lambda^{A} \star z\right)=\chi(\lambda) q(z)$.

Let us now look at the specific case of a scaling. Then

$$
p(z)=\sum_{w \in \mathbb{Z}^{n}} a_{w} z^{w} \Rightarrow p\left(\lambda^{A} \star z\right)=\sum_{w \in \mathbb{Z}^{n}} a_{w} \lambda^{A w} z^{w} .
$$

For $p\left(\lambda^{A} \star z\right)$ to factor as $\chi(\lambda) p(z)$ we must have $A w=A u$ for any two vectors $u, w \in \mathbb{Z}^{n}$ with $a_{v}$ and $a_{u}$ in the support of $p$. Let us fix $u$. Then $w-u \in \operatorname{ker} A$ and $\chi(\lambda)=\lambda^{A u}$. From the previous paragraph we have $\sum_{w \in \mathbb{Z}^{n}} b_{w} \lambda^{A w} z^{w}=$ $q\left(\lambda^{A} \star z\right)=\lambda^{A u} q(z)=\lambda^{A u} \sum_{w \in \mathbb{Z}^{n}} b_{w} z^{w}$. Thus $A u=A w$ 
and therefore there exists $v \in \operatorname{ker} A \cap \mathbb{Z}^{n}$ such that $w=u+v$ for all $w$ with $b_{w}$ in the support of $q$.

The set of rational functions on $\mathbb{K}^{n}$ that are invariant under a group action form a subfield of $\mathbb{K}(z)$ and, as such, it is a finitely generated field. In the case of a scaling the generators of this field can be constructed making use only of linear algebra and the representations of rational invariants given in Lemma 4.1.

Theorem 4.2 Let $V=\left[\begin{array}{ll}V_{\mathfrak{i}}, & V_{\mathfrak{n}}\end{array}\right]$ and $W=\left[\begin{array}{l}W_{\mathfrak{u}} \\ W_{\mathfrak{d}}\end{array}\right]$ be unimodular matrices of integers such that $A V=[H, 0]$ is in column Hermite normal form and $W$ is the inverse of $V$. Then the scaling defined by $A$ has the following properties:

(a) The $n-r$ components of $g=\left[z_{1}, \ldots, z_{n}\right]^{V_{\mathfrak{n}}}$ form $a$ generating set of rational invariants;

(b) Any rational invariant can be written in terms of the components of $g$ by substituting $z=\left[z_{1}, \ldots, z_{n}\right]$ by the respective components of $g^{W_{\mathfrak{o}}}$.

Proof. Observe first that the components of $g$ are invariants. Indeed the columns of $V_{\mathfrak{n}} \operatorname{span} \operatorname{ker} A$ and so $\left(\lambda^{A} \star z\right)^{V_{\mathfrak{n}}}=$ $\lambda^{A V_{\mathfrak{n}}} \star z^{V_{\mathfrak{n}}}=z^{V_{\mathfrak{n}}}$. We shall prove that any rational invariant can be rewritten in terms of these components.

Since $V$ and $W$ are inverses of each other we have $I_{n}=$ $V_{\mathfrak{i}} W_{\mathfrak{u}}+V_{\mathfrak{n}} W_{\mathfrak{o}}$. Thus $z=z^{V_{\mathfrak{i}} W_{\mathfrak{u}}+V_{\mathfrak{n}} W_{\mathfrak{d}}}$, where $z=\left[z_{1}, \ldots, z_{n}\right]^{T}$, the vector of degree 1 monomials. More generally, for any $v \in \mathbb{Z}^{n}, z^{v}=z^{\left(V_{\mathfrak{i}} W_{\mathfrak{u}}+V_{\mathfrak{n}} W_{\mathfrak{o}}\right) v}$. If now $v \in \operatorname{ker} A \cap \mathbb{Z}^{n}$ then $z^{v}=z^{V_{n} W_{\mathfrak{d}} v}=g^{W_{d} v}$ since ker $A \subset \operatorname{ker} W_{\mathfrak{u}}$.

The representation given in Lemma 4.1 implies that any $\frac{p}{q} \in \mathbb{K}(z)^{T}$, with $p, q \in \mathbb{K}[z]$ relatively prime, has the form

$$
p(z)=\sum_{v \in \operatorname{ker} A \cap \mathbb{Z}^{n}} a_{v} z^{u+v} \text { and } q(z)=\sum_{v \in \operatorname{ker} A \cap \mathbb{Z}^{n}} b_{v} z^{u+v}
$$

for some $u \in \mathbb{Z}^{n}$. As elements of $\mathbb{K}(z)$, we can rewrite these as

$$
\begin{aligned}
& p(z)=z^{u} \sum_{v \in \operatorname{ker} A \cap \mathbb{Z}^{n}} a_{v}\left(z^{V_{\mathfrak{n}} W_{\mathfrak{o}}}\right)^{v} \\
& q(z)=z^{u} \sum_{v \in \operatorname{ker} A \cap \mathbb{Z}^{n}} b_{v}\left(z^{V_{\mathfrak{n}} W_{\mathfrak{d}}}\right)^{v}
\end{aligned}
$$

and so

$$
\frac{p(z)}{q(z)}=\frac{p\left(z^{V_{\mathfrak{n}} W_{\mathfrak{o}}}\right)}{q\left(z^{V_{\mathfrak{n}} W_{\mathfrak{o}}}\right)}=\frac{p\left(g^{W_{\mathfrak{o}}}\right)}{q\left(g^{W_{\mathfrak{o}}}\right)} .
$$

Both $V$ and $W$ are needed for computing invariants and rewrite rules. Since a $V$ matrix is produced from a matrix of column operations which convert $A$ to column Hermite form, the $W$ matrix can be computed simultaneously with minimal cost by the inverse column operations.
Example 4.3 Consider the scaling defined by $A=\left[\begin{array}{ll}2 & 3\end{array}\right]$. A unimodular multiplier for its Hermite normal form is

$$
V=\left[\begin{array}{rr}
-1 & 3 \\
1 & -2
\end{array}\right] \quad \text { with inverse } \quad W=\left[\begin{array}{ll}
2 & 3 \\
1 & 1
\end{array}\right]
$$

It follows that $g=\frac{x^{3}}{y^{2}}$ is a generating invariant. Any other rational invariant can be written in terms of $g$ with the substitution $x \mapsto g, y \mapsto g$.

Example 4.4 In order to illustrate the simplicity of our method let us return to the example in the introduction.

Consider the $2 \times 5$ matrix $A$ given by

$$
A=\left[\begin{array}{ccccc}
6 & 0 & -4 & 1 & 3 \\
0 & 3 & 1 & -4 & 3
\end{array}\right]
$$

which defines the group action mentioned in the introduction. Thus if $z=\left(z_{1}, z_{2}, z_{3}, z_{4},, z_{5}\right)$ and $\lambda=(\mu, \nu)$ then the group action defined by $A$ is given by

$$
\lambda^{A} \star z=\left(\mu^{6} z_{1}, \nu^{3} z_{2}, \frac{\nu}{\mu^{4}} z_{3}, \frac{\mu}{\nu^{4}} z_{4}, \mu^{3} \nu^{3} z_{5}\right) .
$$

The column Hermite normal form for $A$ is given by

$$
[H, 0]=\left[\begin{array}{lllll}
3 & 2 & 0 & 0 & 0 \\
0 & 1 & 0 & 0 & 0
\end{array}\right]
$$

and the normal unimodular multiplier and its inverse are

$$
V=\left[\begin{array}{rr|rrr}
1 & 1 & 2 & 1 & 0 \\
1 & 0 & -1 & 2 & 0 \\
1 & 1 & 3 & 2 & 1 \\
1 & 0 & 0 & 2 & 1 \\
0 & 0 & 0 & 0 & 1
\end{array}\right], W=\left[\begin{array}{rrrrr}
2 & -2 & -2 & 3 & -1 \\
0 & 3 & 1 & -4 & 3 \\
\hline 0 & -1 & 0 & 1 & -1 \\
-1 & 1 & 1 & -1 & 0 \\
0 & 0 & 0 & 0 & 1
\end{array}\right] .
$$

Here the last 3 rows of $V_{\mathfrak{n}}$ are the pivot rows. A generating set of invariants is given by the components

$$
\left(g_{1}, g_{2}, g_{3}\right)=z^{V_{\mathfrak{n}}}=\left(\frac{z_{1}^{2} z_{3}^{3}}{z_{2}}, \quad z_{1} z_{2}^{2} z_{3}^{2} z_{4}^{2}, \quad z_{3} z_{4} z_{5}\right)
$$

while the rewrite rules are given by

$$
\left(z_{1}, z_{2}, z_{3}, z_{4}, z_{5}\right) \rightarrow g^{W_{\mathfrak{d}}}=\left(\frac{1}{g_{2}}, \frac{g_{2}}{g_{1}}, g_{2}, \frac{g_{1}}{g_{2}}, \frac{g_{3}}{g_{1}}\right) \text {. }
$$

\subsection{Rational section to the orbits}

The fact we can rewrite any invariant in terms of the generating set by a simple substitution actually reflects the existence and intrinsic use of a rational section [7, 8]. And indeed, any unimodular multiplier for the Hermite normal form provides a rational section. The simplest rational sections are uncovered by the normal unimodular multipliers of Theorem 2.3.

An irreducible variety $\mathcal{P} \subset \mathbb{K}^{n}$ is a rational section for the rational action of an affine algebraic group if there exists a nonempty Zariski open subset $\mathcal{Z} \subset \mathbb{K}^{n}$ such that any orbit of the induced action on $\mathcal{Z}$ intersects $\mathcal{P}$ at exactly one point [21, Section 2.5].

Every vector $a \in \mathbb{Z}^{r}$ can be uniquely written as $a=a^{+}-a^{-}$ where $a^{+}$and $a^{-}$are nonnegative and have disjoint support. Their components are:

$\left[a^{+}\right]_{i}=\left\{\begin{array}{cc}a_{i} & \text { if } a_{i} \geq 0 \\ 0 & \text { otherwise }\end{array} \quad\right.$ and $\left[a^{-}\right]_{i}=\left\{\begin{array}{cc}a_{i} & \text { if } a_{i} \leq 0 \\ 0 & \text { otherwise }\end{array}\right.$ 
This can be extended to $r \times n$ matrices by

$$
A^{+}=\left[\left(A_{\cdot, 1}\right)^{+}, \cdots,\left(A_{., n}\right)^{+}\right] \text {and } A^{-}=\left[\left(A_{., 1}\right)^{-}, \cdots,\left(A_{., n}\right)^{-}\right] \text {. }
$$

Theorem 4.5 With the hypotheses of Theorem 4.2, the variety $\mathcal{P}$ of $\left(z^{V_{\mathrm{i}}^{+}}-z^{V_{\mathrm{i}}^{-}}\right): z^{\infty}$ is a rational section for the scaling defined by $A$. The intersection of the orbit of a point $z \in\left(\mathbb{K}^{*}\right)^{n}$ with this section is the point $z^{V_{\mathfrak{n}} W_{\mathfrak{o}}}$.

Proof. The matrix $W_{\mathfrak{o}}$ is full row rank and $W_{\mathfrak{o}} \cdot\left[V_{\mathfrak{n}}, V_{\mathfrak{i}}\right]=$ $\left[I_{n-r}, 0\right]$. By Lemma 2.2 the columns of $V_{\mathrm{i}}$ span the lattice kernel of $W_{\mathfrak{o}}$. Thus the kernel of

$$
\begin{array}{rlr}
\mathbb{K}[z] & \rightarrow \mathbb{K}\left[x, x^{-1}\right] \\
z & \mapsto x^{W_{\mathfrak{o}}}
\end{array}
$$

is the prime (toric) ideal $P=\left(z^{V_{\mathrm{i}}^{+}}-z^{V_{\mathrm{i}}^{-}}\right):\left(z_{1} \ldots z_{n}\right)^{\infty}$ of dimension $r$ [24, Lemma 4.1, 4.2 and 12.2].

Assume $z \in\left(\mathbb{K}^{*}\right)^{n}$. For $\tilde{z}=\lambda^{A} \star z$ to be on the variety $\mathcal{P}$ of $P$ the components of $\tilde{z}^{V_{\mathrm{i}}}$ need to all be equal to 1 . Thus $\lambda^{A V_{\mathrm{i}}}=z^{-V_{\mathrm{i}}}$, that is, $\lambda^{H}=z^{-V_{\mathrm{i}}}$. Because of the triangular structure of $H$ we can always find $\lambda \in\left(\mathbb{K}^{*}\right)^{r}$ satisfying this equation. For any such $\lambda$ we then have $\tilde{z}=$ $\left(\lambda^{A} \star z\right)^{V_{\mathfrak{i}} W_{\mathfrak{u}}+V_{\mathfrak{n}} W_{\mathfrak{d}}}$ since $V_{\mathfrak{i}} W_{\mathfrak{u}}+V_{\mathfrak{n}} W_{\mathfrak{d}}=I_{n}$ and so $\tilde{z}=$ $\lambda^{H W_{\mathfrak{u}}} \star z^{V_{\mathrm{i}} W_{\mathfrak{u}}+V_{\mathfrak{n}} W_{\mathfrak{d}}}=z^{-V_{\mathfrak{i}} W_{\mathfrak{u}}} \star z^{V_{\mathrm{i}} W_{\mathfrak{u}}+V_{\mathfrak{n}} W_{\mathfrak{d}}}=z^{V_{\mathfrak{n}} W_{\mathfrak{o}}}$ by Proposition 3.1. Thus the intersection of the orbit of $z$ with the variety of $P$ exists, is unique and equal to $z^{V_{\mathfrak{n}} W_{\mathfrak{o}}}$.

From this description we deduce that the invariants $z^{V_{\mathbf{n}} W_{\mathrm{o}}}$ are actually the normalized invariants as defined in [8]. As such the rewriting of Theorem 4.2 applies to the more general class of smooth invariants. Furthermore, if the Hermite form of $A$ is $I_{r}$ there is a global moving frame for the group action and $z^{V_{\mathfrak{n}} W_{\mathfrak{o}}}$ correspond to the normalized invariants as originally defined in [5].

Example 4.6 Consider the scaling given by

$$
\left(z_{1}, z_{2}, z_{3}, z_{4}, z_{5}\right) \rightarrow\left(\frac{\eta}{\nu^{3}} z_{1}, \frac{\eta}{\mu} z_{2}, \eta z_{3}, \frac{\nu}{\eta \mu} z_{4}, \frac{\eta \nu}{\mu} z_{5}\right)
$$

an example used to illustrate dimensional analysis in [19]. In this case the matrix of exponents is

$$
A=\left[\begin{array}{rrrrr}
-3 & 1 & 1 & -1 & 1 \\
0 & -1 & 0 & -1 & -2 \\
1 & 0 & 0 & 1 & 1
\end{array}\right]
$$

The normal unimodular multiplier and its inverse are

$V=\left[\begin{array}{rrr|rr}0 & 0 & 1 & -1 & -1 \\ 0 & -1 & 0 & -1 & -2 \\ 1 & 1 & 3 & -1 & -2 \\ 0 & 0 & 0 & 1 & 0 \\ 0 & 0 & 0 & 0 & 1\end{array}\right], W=\left[\begin{array}{rrrrr}-3 & 1 & 1 & -1 & 1 \\ 0 & -1 & 0 & -1 & -2 \\ 1 & 0 & 0 & 1 & 1 \\ \hline 0 & 0 & 0 & 1 & 0 \\ 0 & 0 & 0 & 0 & 1\end{array}\right]$.

Thus the rewrite rules are simply $z \rightarrow g^{W_{\mathfrak{d}}}=\left(1,1,1, g_{1}, g_{2}\right)$. By Theorem 4.5 the associated rational section is the variety $\left(z_{3}-1, z_{3}-z_{2}, z_{3}^{3}-z_{1}\right): z^{\infty}$. Simple combinations of the ideal generators show that this ideal is equal to $\left(z_{1}-1, z_{2}-\right.$ $\left.1, z_{3}-1\right)$
Example 4.3 illustrates a case where things are particularly simple. Namely, the simplest case for the normalization of the unimodular multiplier $V$ occurs when the pivot rows of $V_{\mathfrak{n}}$ are the rows of an $(n-r)$-identity matrix. Assuming that the pivot rows appear at the end, a situation that can be arranged by permuting the columns of $A$ and therefore the order of the original variables, then the normal unimodular multiplier and its inverse are

$$
V=\left[\begin{array}{cc}
V_{\mathrm{i}}^{*} & V_{\mathfrak{n}}^{*} \\
0 & I_{n-r}
\end{array}\right] \text { and } W=\left[\begin{array}{cc}
V_{\mathrm{i}}^{*-1} & -V_{\mathrm{i}}^{*-1} V_{\mathfrak{n}}^{*} \\
0 & I_{n-r}
\end{array}\right] .
$$

The rewrite rules are then: $z \rightarrow g^{W_{\mathfrak{d}}}=\left(1, \ldots, 1, g_{1}, \ldots, g_{n-r}\right)$ which indicates that the equations for the section can be made simpler than in Theorem 4.5.

Proposition 4.7 If the canonical unimodular multiplier $V$ of $A$ for its Hermite normal form is

$$
V=\left[\begin{array}{cc}
V_{\mathrm{i}}^{*} & V_{\mathfrak{n}}^{*} \\
0 & I_{n-r}
\end{array}\right]
$$

then the variety of $\left(z_{1}-1, \ldots, z_{r}-1\right)$ is a rational section to the scaling defined by $A$. There are then $n-r$ generating invariants $g_{r+1}^{*}, \ldots, g_{n}^{*}$ s.t. any other rational invariants can be written in terms of those with the substitution $\left(z_{1}, \ldots, z_{n}\right) \mapsto\left(1, \ldots, 1, g_{r+1}^{*}, \ldots, g_{n}^{*}\right)$.

The proof proceeds by taking the power $\left(V_{i}^{*}\right)^{-1}$ of $\left(z^{V_{i}^{+}}-\right.$ $\left.z^{V_{i}^{-}}\right)$. The components then belong to the ideal generated by the components of $\left(z^{V_{\mathrm{i}}^{+}}-z^{V_{\mathrm{i}}^{-}}\right)$and factor as a product of $\left(z-1_{n}\right)$ with a monomial in $z$. Then $\left(1, \ldots, 1, g_{r+1}^{*}, \ldots, g_{n}^{*}\right)=$ $z^{V_{\mathfrak{n}} \cdot W_{\mathfrak{o}}}=\left(1_{r}, z^{V_{\mathfrak{n}}}\right)=\left(1_{r}, g\right)$.

Note that the form (7) is the only possibility for the $n-r$ bottom rows of $V_{\mathrm{i}}$ to be zero. Indeed, since this implies that the $n-r$ bottom rows of $V_{\mathfrak{n}}$ form a unimodular matrix, and, since it is in Hermite form, it can only be the identity.

\section{REDUCING POLYNOMIAL SYSTEMS}

If the solution set of a polynomial system of equations is invariant under a group action, then there is an equivalent system given in terms of invariants of this group action [19]. The equivalent system written in terms of a generating set of invariants is the reduced system. However, for general symmetry reductions a further problem is to recover the solutions of the original system from the solutions of the reduced system.

In this section we show how to fully work out a symmetry reduction for a scaling symmetry. If the scaling symmetry is $r$ dimensional, then the reduced system has $r$ fewer variables. In addition, we show how to retrieve all toric solutions of the original system from the toric solutions of the reduced system. We shall indeed discount the solutions for which there is a zero component. This is a relevant case. For instance, in a chemical reaction or a population dynamics model we look for the equilibria where no species disappears.

We consider a set of equations $p_{1}(z)=0, \quad \ldots, \quad p_{m}(z)=0$ where $p_{1}, \ldots, p_{m}$ are in $\mathbb{K}[z]=\mathbb{K}\left[z_{1}, \ldots, z_{n}\right]$ or even in the Laurent polynomial ring $\mathbb{K}\left[z, z^{-1}\right]$ since we are concerned with solutions in $\left(\mathbb{K}^{*}\right)^{n}$. For convenience we introduce the 
map $p=\left(p_{1}, \ldots, p_{m}\right)$ and write the system of equations as $p(z)=0$.

Definition 5.1 The matrix $A \in \mathbb{Z}^{r \times n}$ defines a scaling symmetry for the polynomial system $p(z)=0$ if, for a given $z \in\left(\mathbb{K}^{*}\right)^{n}$, we have

$$
p(z)=0 \Rightarrow p\left(\lambda^{A} \star z\right)=0, \quad \forall \lambda \in\left(\mathbb{K}^{*}\right)^{r} .
$$

In the following we suppose that $A \in \mathbb{Z}^{r \times n}$ defines a scaling symmetry for the polynomial system $p(z)=0$. Appendix A provides a way of determining some of these symmetries. Then $V$ is a unimodular multiplier such that $A \cdot V$ is the Hermite normal form of $A$, and $W$ is the inverse of $V$. The invariantization of $p \in \mathbb{K}\left[z, z^{-1}\right]$ associated to a choice of $V$ is a Laurent polynomial $q$ in $n-r$ variables $\left(y_{1}, \ldots, y_{n-r}\right)$ defined by $q(y)=f\left(y^{W d}\right)$. From Theorem 4.2 we know that if $p$ is invariant then $p(z)=q(g)$ where $g=z^{V_{\mathfrak{n}}}$. Yet we do not restrict invariantization to invariants as there is no need for the polynomials defining the symmetric system to be invariant.

Proposition 5.2 Let $q_{1}, \ldots, q_{m}$ in $\mathbb{K}\left[y, y^{-1}\right]$ be defined as $q_{i}(y)=p_{i}\left(y^{W d}\right)$. If $y \in\left(\mathbb{K}^{*}\right)^{n-r}$ is a solution of $q(y)=0$, then for all $\lambda \in\left(\mathbb{K}^{*}\right)^{r}, \lambda^{A} \star y^{W_{\mathfrak{o}}}$ is a solution of $p(z)=0$.

The Laurent polynomials $q_{1}, \ldots, q_{m}$ form the reduced system. This reduced system has $r$ fewer variables than the original system. As described in the above proposition, any point on its solution set provides a parameterized $r$ dimensional set of solutions for the original system. Proposition 5.2 is an immediate result of the symmetry condition (8). The following result is a stronger assertion: any toric solution of the original system can be obtained that way.

Theorem 5.3 Assume that $A \in \mathbb{Z}^{r \times n}$ defines a scaling symmetry for the polynomial system $p(z)=0$ and that $q(y)=0$ is the reduced system. Then for any $z \in\left(\mathbb{K}^{*}\right)^{n}$ satisfying $p(z)=0$ there exists $\lambda \in\left(\mathbb{K}^{*}\right)^{r}$ and $y \in\left(\mathbb{K}^{*}\right)^{n-r}$ such that $q(y)=0$ and $z=\lambda^{A} \star y^{W_{\mathfrak{o}}}$.

Proof. Assume $z \in\left(\mathbb{K}^{*}\right)^{n}$ satisfies $p(z)=0$. Since $H$ is triangular and nonsingular, there exists $\lambda \in\left(\mathbb{K}^{*}\right)^{r}$ such that $\lambda^{H}=z^{-V_{\mathrm{i}}}$. Set $y=z^{V_{\mathrm{n}}}$. Since $\lambda^{[H, 0]}=\left(z^{-V_{\mathrm{i}}}, 1_{n-r}\right)$ we have

$$
\left(\lambda^{A} \star z\right)^{\left[V_{\mathfrak{i}}, V_{\mathfrak{n}}\right]}=\lambda^{[H, 0]} \star\left(z^{V_{\mathfrak{i}}}, z^{V_{\mathfrak{n}}}\right)=\left(1_{r}, y\right) .
$$

Taking both sides of the above equality to the power $W$ gives

$$
\lambda^{A} \star z=\left(\lambda^{A} \star z\right)^{V \cdot W}=\left(1_{r}, y\right)^{\left[\begin{array}{l}
W_{\mathfrak{u}} \\
W_{\mathfrak{o}}
\end{array}\right]}=y^{W_{\mathfrak{o}}} .
$$

By the symmetry hypothesis $p\left(y^{W_{\mathfrak{d}}}\right)=p\left(\lambda^{A} \star z\right)=0$. Thus $q(y)=0$.

There is a geometric interpretation for the above approach that stems out of the work of $[5,7,8]$. Namely, the solution set of the reduced system describes the projection, along the orbits, of the original solution set on the section $z^{V_{i}}=1$. From the above proof it is clear that the group element $\lambda \in$ $\left(\mathbb{K}^{*}\right)^{r}$ providing the link between the solution of the original system and the solution of the reduced system is unique if and only if the Hermite normal form is the identity.

Example 5.4 Consider the system of polynomial equations

$$
\begin{aligned}
z_{2} z_{4}{ }^{2}-z_{1} & =0 \\
z_{1} z_{3}-z_{2} & =0 .
\end{aligned}
$$

presented in [14, Example 3.14]. On one hand we can look for the solutions that have a zero component. They are part of the two-parameter family of solutions given by $(0,0, \alpha, \beta)$. On the other hand we can determine a scaling symmetry for this system with the method of Appendix A which gives

$$
A=\left[\begin{array}{cccc}
1 & 1 & 0 & 0 \\
0 & 2 & 2 & -1
\end{array}\right] .
$$

$A$ unimodular multiplier $V$, and its inverse $W$, to obtain the Hermite normal form of $A$ are

$$
V=\left[\begin{array}{rr|rr}
1 & -1 & 1 & -1 \\
0 & 1 & -1 & 1 \\
0 & 0 & 1 & 0 \\
0 & 1 & 0 & 2
\end{array}\right], W=\left[\begin{array}{rrrr}
1 & 1 & 0 & 0 \\
0 & 2 & 2 & -1 \\
\hline 0 & 0 & 1 & 0 \\
0 & -1 & -1 & 1
\end{array}\right]
$$

The reduced system is thus obtained by substituting $\left(z_{1}, z_{2}, z_{3}, z_{4}\right)$ by $\left(y_{1}, y_{2}\right)^{W_{\mathfrak{o}}}=\left(1, \frac{1}{y_{2}}, \frac{y_{1}}{y_{2}}, y_{2}\right)$ :

$$
\left.\begin{array}{l}
\frac{1}{y_{2}} y_{2}^{2}-1=0 \\
\frac{y_{1}}{y_{2}}-\frac{1}{y_{2}}=0
\end{array}\right\} \Leftrightarrow\left\{\begin{array}{l}
y_{1}=1 \\
y_{2}=1 .
\end{array}\right.
$$

The latter system has a solution set consisting of a single point. It provides a two parameter solution to the original system: $\lambda^{A} \star(1,1)^{W_{\mathfrak{d}}}=\left(\lambda_{1}, \lambda_{1} \lambda_{2}^{2}, \lambda_{2}^{2}, \lambda_{2}^{-1}\right)$. By Theorem 5.3, any solution, without zero component, of the original system is obtained in this way. Since $A \cdot V=\left[\begin{array}{ll}I_{2} & 0\end{array}\right]$, the pair $\left(\lambda_{1}, \lambda_{2}\right)$ providing the given solution is unique. It can be read from the columns of $V_{\mathrm{i}}:\left(\lambda_{1}, \lambda_{2}\right)=\left(\frac{1}{z_{1}}, \frac{z_{1}}{z_{2} z_{4}}\right)$.

For the geometric interpretation note that the underlying rational section is the variety of $\left(z_{1}-1, z_{2} z_{4}-z_{1}\right)$. One can check that the intersection of the solution set of the original system with this section is $(1,1)^{W_{\mathfrak{d}}}=(1,1,1,1)$. Any element in the orbit of this point solves the original system.

The semi-rectified system obtained in [14] is different than our reduced system. The process described there introduces square roots and the semi-rectified system has two solutions. This owes to the row-echelon form used. In our approach we get a clear connection between the toric solutions of the reduced system and of the original system. As we are free of fractional powers, we avoid having to pay attention to the sign of the components in the solution set.

Example 5.5 Consider the polynomial system of 3 equations in 5 variables given by

$$
\begin{aligned}
& z_{1}^{4} z_{3}^{6}-5 z_{1}^{2} z_{2} z_{3}^{3}+6 z_{2}^{2}=0 \\
& z_{1}^{2} z_{2}^{5} z_{3}^{4} z_{4}^{4}-2 z_{1}^{3} z_{2}^{2} z_{3}^{5} z_{4}^{2}-z_{1}^{2} z_{3}^{3}+z_{2}=0 \\
& z_{1} z_{2}^{3} z_{3}^{4} z_{4}^{3} z_{5}-z_{1}^{2} z_{3}^{3}-z_{2}=0 .
\end{aligned}
$$


On one hand there is a three-parameter family of solutions given by $(0,0, \alpha, \beta, \gamma)$. On the other hand, a symmetry of this system is given by the $2 \times 5$ matrix $A$ of Example 4.4 . The reduced system

$$
\begin{array}{r}
y_{1}^{2}-5 y_{1}+6=0 \\
y_{2}^{2}-2 y_{1} y_{2}+y_{1}+1=0 \\
y_{2} y_{3}-y_{1}-1=0 .
\end{array}
$$

is obtained with the substitution:

$$
\left(z_{1}, z_{2}, z_{3}, z_{4}, z_{5}\right) \mapsto\left(\frac{1}{y_{2}}, \frac{y_{2}}{y_{1}}, y_{2}, \frac{y_{1}}{y_{2}}, \frac{y_{3}}{y_{1}}\right) .
$$

The solution set of the above reduced system consists of the 4 points $(2,1,3),(2,3,1),(3,3+\sqrt{5}, 3-\sqrt{5})$ and $(3,3-\sqrt{5}, 3+$ $\sqrt{5})$. In this case the underlying rational section is the variety of $\left(z_{1} z_{2} z_{3} z_{4}-1, z_{2} z_{4}-1\right)$. The intersection of the solution set of the original system are the four points $(2,1,3)^{W_{\mathfrak{d}}}=$ $\left(1, \frac{1}{2}, 1,2, \frac{3}{2}\right),(2,3,1)^{W_{\mathfrak{o}}}=\left(\frac{1}{3}, 3,3, \frac{1}{3}, \frac{1}{2}\right),(3,3+\sqrt{5}, 3-\sqrt{5})^{W_{\mathrm{o}}}$ and $(3,3-\sqrt{5}, 3+\sqrt{5})^{W_{\mathrm{o}}}$. Any element in the orbits of these points is a solution of the original system. We thus have four parameterized two dimensional solution subsets. For example, $\lambda^{A} \star(2,1,3)^{W_{\mathfrak{o}}}=\left(\mu^{6}, \frac{\nu^{3}}{2}, \frac{\nu}{\mu^{4}}, \frac{2 \nu}{\mu^{4}}, \frac{3 \mu^{3} \nu^{3}}{2}\right)$ is a parameterized two-dimensional subset of solutions. By Theorem 5.3, all solutions, without zero component, of the original system are obtained in this way.

\section{CONCLUSION}

In this paper we have made use of the Hermite Normal Form of the matrix of exponents of a scaling symmetry. Invariants, rewrite rules and rational section for a scaling are all determined from an associated unmimodular multiplier and its inverse. We have also illustrated how scaling can be used to reduce polynomial systems of equations. All the algorithms in this paper have been implemented in the computer algebra system Maple with the code available from the authors.

There are a number of research topics that follow from our work. The Hermite Normal Form is not the only rankrevealing or normalizing transformation of an integer matrix. Other possibilities include using the Smith Normal Form of the scaling matrix or lattice reduction basis (i.e. LLL) for the normal unimodular multiplier. We are interested in the invariants, rewrite rules and sections that result from using these alternate forms, in particular seeing when these are simpler than those that result from the use of the Hermite form.

We have shown how to reduce polynomial systems of equations by scaling symmetries. This can be extended to dynamical systems and, more generally, to the reduction of systems of differential and algebraic equations. We intend to report on our progress with this in a future publication.

\section{REFERENCES}

[1] B. Beckermann, G. Labahn, and G. Villard. Normal forms for general polynomial matrices. Journal of Symbolic Computation, 41(6):708-737, 2006.

[2] G. Birkhoff. Hydrodynamics: A study in logic, fact and similitude. Princeton Univ. Press, 1960.

[3] P. Bridgman. Dimensional Analysis. Yale Univ. Press, 1931.
[4] H. Cohen. A course in computational algebraic number theory. Springer-Verlag, 1993.

[5] M. Fels and P. Olver. Moving coframes. ii. Regularization and theoretical foundations. Acta Appl. Math., 55(2):127-208, 1999.

[6] G. Havas, B. Majewski, and K. Matthews. Extended gcd and Hermite normal form algorithms via lattice basis reduction. Experimental Mathematics, 7(2), 1998.

[7] E. Hubert and I. Kogan. Rational invariants of a group action. Construction and rewriting. Journal of Symbolic Computation, 42(1-2):203-217, 2007.

[8] E. Hubert and I. Kogan. Smooth and algebraic invariants of a group action. Local and global constructions. Foundations of Computational Mathematics, 7(4), 2007.

[9] E. Hubert and A. Sedoglavic. Polynomial Time Nondimensionalisation of Ordinary Differential Equations via their Lie Point Symmetries, 2006.

[10] H. E. Huntley. Dimensional Analysis. Dover Publications, New York, 1967.

[11] Y. Ishida. Formula processing on physical systems. Complex Systems, 11(2):141-160, 1997.

[12] G. Kemper. The computation of invariant fields and a new proof of a theorem by Rosenlicht. Transformation Groups, 12:657-670, 2007.

[13] R. Khanin. Dimensional Analysis in Computer Algebra. In Proceedings of ISSAC. ACM press, 2001.

[14] F. Lemaire and A. Ürgüplü. A method for semi-rectifying algebraic and differential systems using scaling type Lie point symmetries with linear algebra. In Proceedings of ISSAC. ACM press, 2010.

[15] C. Lin and L. Segel. Mathematics applied to deterministic problems in the natural sciences. Society for Industrial and Applied Mathematics, 1988.

[16] E. Mansfield. A Practical Guide to the Invariant Calculus. Cambridge University Press, 2010.

[17] J. Müller-Quade and T. Beth. Calculating generators for invariant fields of linear algebraic groups. In Applied algebra, algebraic algorithms and error-correcting codes, volume 1719 of LNCS. Springer, 1999.

[18] J. D. Murray. Mathematical Biology, volume 17 of Interdisciplinary Applied Mathematics. Springer, 2002.

[19] P. J. Olver. Applications of Lie Groups to Differential Equations. Number 107 in Graduate texts in Mathematics. Springer-Verlag, New York, 1986.

[20] A. Parshin and I. Shafarevich, editors. Algebraic Geometry. IV, volume 55 of Encyclopaedia of Mathematical Sciences. Springer-Verlag, 1994.

[21] V. L. Popov and E. B. Vinberg. Invariant Theory. In Algebraic geometry. IV, Encyclopaedia of Mathematical Sciences. Springer-Verlag, 1994.

[22] A. Schrijver. Theory of Linear and Integer Programming. Wiley, 1986.

[23] A. Sedoglavic. Reduction of algebraic parametric systems by rectification of their affine expanded lie symmetries. In Algebraic Biology, volume 4545 of LNCS. Springer, 2007.

[24] B. Sturmfels. Gröbner bases and convex polytopes. American Mathematical Society, Providence, RI, 1996. 


\section{APPENDIX}

\section{A. FINDING SCALING SYMMETRY OF A POLYNOMIAL SYSTEM}

Suppose we have $m$ equations of the form

$$
p_{k}(z) \equiv c_{k, 0} z^{\alpha_{k, 0}}+c_{k, 1} z^{\alpha_{k, 1}}+\cdots+c_{k, \ell_{k}} z^{\alpha_{k, \ell_{k}}}=0
$$

where $z=\left(z_{1}, \ldots, z_{n}\right)$. A sufficient condition for the solution set of this system to have a given group action as a symmetry is that the polynomials are semi-invariant for this group action. In the case of a scaling determined by a integer matrix $A$ this condition is that $p_{k}(z)$ divides $p_{k}\left(\lambda^{A} \star z\right)$. As we address only toric solutions, that is, solutions where no component is zero, we can work with Laurent polynomials and normalize all the equations by dividing out the first monomial. We get equations of the form

$$
q_{k}(z) \equiv c_{k, 0}+c_{k, 1} z^{\beta_{k, 1}}+\cdots+c_{k, \ell_{k}} z^{\beta_{k, \ell_{k}}}=0
$$

where $\beta_{k, j}=\alpha_{k, j}-\alpha_{k, 0}$ for all $k$ and all $j>0$. The sufficient condition for the solution set to have a scaling symmetry is then that the Laurent polynomials $q_{1}, \ldots, q_{m}$ are invariants. Since

$q_{k}\left(\lambda^{A} \star z\right)=c_{k, 0}+c_{k, 1} \lambda^{A \beta_{k, 1}} z^{\beta_{k, 1}}+\cdots+c_{k, \ell_{k}} \lambda^{A \beta_{k, \ell_{k}}} z^{\beta_{k, \ell_{k}}}$

The invariance condition $q_{k}\left(\lambda^{A} \star z\right)=q_{k}(z)$ results in $\lambda^{A \beta_{k, 1}}=$ $\cdots=\lambda^{A \beta_{k, \ell_{k}}}=1$ for all $k$. It implies that each $\beta_{k, j}$ is in the kernel of $A$. Thus $A$ is the matrix having kernel

$$
K=\left[\beta_{1,1}, \ldots, \beta_{m, \ell_{m}}\right] .
$$

In order to determine $A$ let us assume that we have a matrix $K$ and we are looking for a matrix $A$ such that $A \cdot K=0$ with $A$ of full row rank. That is, we look for a basis of the left nullspace of $K$, as a module over the integers.

If $K$ has full column rank and we take transposes then we recognize the problem as one solved in Lemma 2.2. If $K$ is not of full column rank then we can still reduce the problem via the technique described at the end of Subsection 3.2 (again taking transposes).

Thus one method to find left nullspace bases (after ensuring that $K$ is of full column rank) is to use unimodular row operations $U$ to determine

$$
U \cdot K=\left[\begin{array}{c}
H \\
0
\end{array}\right]
$$

with $H$ of full row rank. If there are $r$ rows of zeros on the right term in (10) then we can let $A$, the matrix of the scaling, be the last $r$ rows of $U$. Note that, since $U$ is unimodular, the resulting matrix $A$ is also of full row rank.

Example A.1 Suppose $K$ is given by

$$
K=\left[\begin{array}{rr}
-1 & 1 \\
1 & -1 \\
0 & 1 \\
2 & 0
\end{array}\right]
$$

the kernel which comes from the polynomial system of equations in Example 5.4. Then a unimodular matrix which re- veals the rank of this matrix is given by

$$
\left[\begin{array}{rrrr}
0 & -1 & -1 & 0 \\
-1 & -1 & -1 & 0 \\
1 & 1 & 0 & 0 \\
0 & 2 & 2 & -1
\end{array}\right]\left[\begin{array}{rr}
-1 & 1 \\
1 & -1 \\
0 & 1 \\
2 & 0
\end{array}\right]=\left[\begin{array}{rr}
-1 & 0 \\
0 & -1 \\
0 & 0 \\
0 & 0
\end{array}\right] \text {. }
$$

A scaling matrix is thus

$$
A=\left[\begin{array}{rrrr}
1 & 1 & 0 & 0 \\
0 & 2 & 2 & -1
\end{array}\right] .
$$

The alternate scaling used in [14] when treating Example 5.4 is

$$
A^{\prime}=\left[\begin{array}{rrrr}
-2 & 0 & 2 & -1 \\
0 & -2 & -2 & 1
\end{array}\right]
$$

In this case $A^{\prime}=U \cdot A$ where

$$
U=\left[\begin{array}{rr}
-2 & 1 \\
0 & -1
\end{array}\right]
$$

and, since $U$ is not unimodular, the action of the scaling described by $A^{\prime}$ has nontrivial isotropy.

When using a Hermite reduction, the rows of $A$ form a basis for the integer lattice of the left kernel of $K$. Any other integer matrix $A^{\prime}$ whose rows are in the left kernel of $K$ are of the form $A^{\prime}=U \cdot A$. The rows of $A^{\prime}$ then forms a basis for the left kernel of $K$ if and only if $U$ is unimodular. 